\title{
Salivary Levels of ErbB2 and CEA in Oral Squamous Cell Carcinoma Patients
}

\author{
Marieh Honarmand ${ }^{1}$, Leila Farhad-Mollashahi ${ }^{1 *}$, Alireza Nakhaee ${ }^{2}$, Masoume \\ $\mathrm{Nehi}^{3}$
}

\begin{abstract}
Oral squamous cell carcinoma (OSCC) is one of the ten most common causes of cancer death worldwide. Assessment of molecular changes can help detect and control lesions. The aim of this study was evaluation of salivary levels of ErbB2 and CEA in OSCC patients. In this case-control study, 27 OSCC patients and 26 healthy controls participated. After obtaining consent and filling out a questionnaire, unstimulated saliva samples were collected from people in the morning for measurement of the two markers using ELISA. Data were analyzed using Mann Whitney U test in SPSS 19 software with $\mathrm{P}<0.05$ considered significant. Subjects were in the age range of 40 to 65 years. ISalivary level CEA was elevated in OSCC samples $(42.6 \pm 21.1 \mathrm{ng} / \mathrm{ml})$ as compared to those of controls $(22.6 \pm 22.1 \mathrm{ng} / \mathrm{ml})(\mathrm{p}<0.01)$, but no significant variation was noted for ErbB2 $(5.2 \pm 1.8 \mathrm{ng} / \mathrm{ml}$ and $4.93 \pm 2.14 \mathrm{ng} / \mathrm{ml}, \mathrm{p}=0.28)$. The role of ErbB2 as a tumor marker in patients with OSCC must still be regarded as controversial and needs further studies to clarify any significance for early detection or screening. In contrast the salivary level of CEA may find application for early detection of patients.
\end{abstract}

Keywords: Oral squamous cell carcinoma - ErbB2 - CEA - salivary samples

Asian Pac J Cancer Prev, 17, Cancer Control in Western Asia Special Issue, 77-80

\section{Introduction}

Oral squamous cell carcinoma (OSCC) is the most common cancer of the mouth and constitutes about $90 \%$ of oral cavity cancers. Approximately $95 \%$ of all OSCCs occur in patients older than 40 years with a mean age of 60 years (Greenberg et al., 2008).

The use of saliva is developing as a non-invasive, cost-effective diagnostic tool for screening of patients, without any need for advanced approaches (Bonne et al., 2012; Rafael et al., 2006). Biomarkers are useful, changeable substances in saliva and their levels vary during health and sickness and can be used for diagnosis and follow-up of certain diseases such as oral and breast cancers, and periodontal diseases (Liu et al., 2012).

Epidermal growth factor receptors (EGFRs), also known as Her and erbB1, were the first group to be identified in the family erbB receptors, which belong to the bigger family of tyrosine kinases (Sebastian et al., 2006, Normanno et al., 2006). Other members of this family, including Her2 (erbB2), Her3 (erbB3), and Her4 (erbB4), were identified later. Activity of these receptors is necessary for normal growth and cellular differentiation through ATP required by cells (Rautava et al., 2008).

EGFR is associated with many malignancies. This receptor is active in cancers and increases cell proliferation. Therefore, blockers of this receptor are used in cancer treatment (Soulieres et al., 2004).

ErbB2 is a proto-oncogene located on chromosome $17 q$ and encodes the tyrosine kinase growth factor. Measuring the level of erbB2 protein is a reliable method for the initial diagnosis and screening of cancers (Streckfus et al., 2004).

Carcinoembryonic antigen (CEA) is a glycoprotein involved in cellular adhesion, which has been evaluated in many malignancies, and its levels reflect the progress or regression of the disease, which is useful for the initial diagnosis, relapse, and treatment control of cancers (Boehm et al., 2000; Bo et al., 2015). High serum levels of CEA are seen in patients with colon carcinoma or GI tract carcinoma (Yakabe et al., 2010).

Since few studies have been performed on these two biomarkers, especially in Iran, the present study aimed at comparing the levels of erbB2 and CEA in the saliva of patients with oral cancer and healthy people.

${ }^{1}$ Oral and Dental Disease Research Center, Department of Oral Medicine, School of Dentistry, ${ }^{2}$ Department of Biochemistry, School of Medicine, ${ }^{3}$ Department of Oral Medicine, Dental School, Zahedan University of Medical Sciences, Zahedan, Iran . *For Correspondence: Im farhad.zaums@yahoo.com 


\section{Materials and Methods}

The study population consisted of 27 patients with OSCC and 26 controls that were referred to the Oral

Disease Department of Zahedan Dentistry School from 2013 to 2014. All subjects were informed about the research and agreed to participate on the study and filled informed consent forms. A questionnaire was prepared containing the information and medical history. Exclusion criteria were: history of Sjogren's syndrome, radiotherapy, consumption of drugs effective on saliva, other oral and dental afflictions, and any systemic or inflammatory disease. The groups were matched in terms of age and gender. Unstimulated saliva was collected in the morning (the best time for saliva collection). To avoid any stimulation, the patients were asked to refrain from eating, drinking or smoking 90 minutes before the test. Each subject ejected his/her saliva into a tube every 60 seconds for 2-5 minutes $(1 \mathrm{~mL})$. The saliva was then centrifuged at $10,000 \mathrm{rpm}$ at $4{ }^{\circ} \mathrm{C}$ for 5 minutes to separate cellular and bacterial debris, and the saliva was stored at $-20{ }^{\circ} \mathrm{C}$ for later analyses.

ErbB2 and CEA were measured by ELISA test using Eastbiopharm (No: ck-E91175, China) and Monobind (No: 1825-300, USA) kits, respectively, according to manufacturers' protocols. A standard curve was plotted and the samples' concentrations were calculated using this curve. All samples were measured in duplicates.

The concentrations of erbB2 and CEA were expressed in $\mathrm{ng} / \mathrm{mL}$. The data obtained from the study was described using (absolute and relative) frequency tables and the Mann-Whitney test to analyze them using SPSS-19. Significant level for tests was 0.05 .

\section{Results}

The levels of erbB2 and CEA were measured in saliva samples of 27 patients with OSCC and of 26 subjects in the control group. In OSCC group, respectively, $11(40.7 \%), 7(25.9 \%)$ and $9(33.4 \%)$ patients were in stage 1,2 and 3 . None of the variables was normally distributed.Therefore, the Mann-Whitney was used to compare

Table1. Demographics and Salivary Concentrations of Biomarkers in Study Groups

\begin{tabular}{lccc}
\hline Characteristics & Case Group & Control Group & p-value \\
\hline Age & $53.8 \pm 5.9$ & $52.8 \pm 7.4$ & 0.4 \\
(male/female) & $15.0 / 12.0$ & $14.0 / 12.0$ & 0.9 \\
Gender & & & \\
(ng/ml)CEA & $42.6 \pm 21.1$ & $22.6 \pm 22.1$ & 0.0 \\
(ng/ml)Erb B2 & $5.2 \pm 1.8$ & $4.9 \pm 2.1$ & 0.3 \\
\hline *Mann- Whitney U Test
\end{tabular}

Table 2. Salivary Concentrations of Biomarkers by Gender

\begin{tabular}{|c|c|c|c|c|c|c|}
\hline \multirow{2}{*}{ Characteristics } & \multicolumn{3}{|c|}{ Case Group } & \multicolumn{3}{|c|}{ Control Group } \\
\hline & $\operatorname{Male}(\operatorname{mean} \pm \mathrm{SD})$ & Female $($ mean $\pm S D)$ & p-value $e^{*}$ & $\operatorname{Male}($ mean $\pm S D)$ & Female $($ mean $\pm S D)$ & p-value ${ }^{*}$ \\
\hline (ng/ml)CEA & $42.9 \pm 22.8$ & $42.2 \pm 19.7$ & 0.9 & $28.8 \pm 22.9$ & $19.3 \pm 15.3$ & 0.1 \\
\hline (ng/ml)Erb B2 & $5.6 \pm 1.5$ & $4.6 \pm 2.1$ & 0.2 & $4.9 \pm 2.5$ & $4.9 \pm 1.8$ & 0.8 \\
\hline
\end{tabular}

them in the two groups. The levels of CEA increased in the case group compared to the control group;however, no significant difference was observed in the levels of erbB2 between the two groups. In addition, there were no significant differences between male and female patients with respect to the CEA and ErbB2 tumor markers. Information regarding description and analysis of CEA and erbB2 concentrations and their relationships with gender and age are shown in Tables 1 and 2.

\section{Discussion}

The study population consisted of 27 patients with oral squamous cell carcinoma (case group) and 26 healthy subjects (control group). The subjects were in the age range of 40-65 years. CEA levels increased in OSCC samples, but the erbB2 biomarker levels in the patient and control groups were not significantly different. Moreover, no significant gender difference existed in the levels of these two markers.

Oral squamous cell carcinoma is one of the sixth most common cancer in Asia and is associated with a high mortality rate. Aside from the importance of prevention, early diagnosis is a vital determinant of successful treatment, improved survival rate of cancer patients (Greenberg et al., 2008).

$\mathrm{ErbB} 2$ is proto-oncogene located on chromosome 17q that encodes the tyrosine kinase growth factor receptor. Determining the levels of erbB2 protein is a reliable method for early diagnosis and screening of cancer (Streckfus et al., 2004).

Bayrak et al (2013) found that overexpression of genes is seen in many solid tumors, which is useful in disease prognosis and response to treatment. Their results showed that overexpression of erbB2 can be a strong prognostic factor in patients with metastatic gastric cancer.

In a study in Brazil, Da Saliva et al. examined erbB2 expression in OSCC tissue samples. They found a strong positive correlation between high expression of erbB2 and OSCC. In addition, expression of erbB2 was associated with perineural infiltration, lymph node metastasis, increased recurrence, and poor prognosis of the disease (Da saliva et al., 2009).

Chen et al. conducted a study on patients with oral cancer. They examined the expression level of Her2/ neu in serums of patients with OSCC and reported that overexpression of neu/Her2 was associated with advanced disease, metastasis, and clinical complications. These researchers found a distinct correlation between $\mathrm{Her} 2 /$ neu expression and metastasis to lymph nodes. After treatment, levels of Her $2 /$ neu declined considerably. The researchers believed the serum level of Her2/neu was a useful marker for assessing the effectiveness of treatment, control of SCC

*Mann- Whitney U Test 
recurrence, and survival rate in patients with oral cancer (Chen et al., 2007).

In a study conducted, Chopra et al. examined serum and salivary levels of Her2/neu in premalignant and malignant lesions. They showed there were no significant differences between the groups with respect to serum levels of Her2/neu, but the salivary Her2/neu level was significantly higher in patients compared to healthy subjects. Moreover, the salivary Her2/neu level in malignant lesions was significantly different from that in pre-malignant lesions. These researchers suggested that Her2/neu could be a useful marker for differentiating premalignant from malignant lesions (Chopra et al., 2015).

The results of these studies are inconsistent with those of our study. This difference may be due to differences in the method of sample collection, sample type, and the method of determining erbB2 levels.

In a study in Shiraz, Sardary et al. evaluated Her2/neu expression in squamous cell carcinoma of the head and neck. They examined 28 patients with SCC of the head and neck, and analyzed Her2/neu expression in saliva. These researchers reported that the salivary levels of Her2/neu were not higher in patients with head and neck SCC compared to the control group (Sardary et al., 2012).

Angiero et al. used immunohistochemical methods to study Her2/neu expression in 40 tissue samples of patients with tongue cancer. No relationship was observed between the levels of Her2/neu and other clinical-pathological parameters or prognostic factors. They believed Her2/neu couldn't a primary role in clinical control and treatment of SCC of the tongue (Angiero et al., 2008).

In a study Ulanovski et al. examined erbB2 expression in 27 patients with SCC of the tongue. They reported that elevated levels of erbB2 could not be used as a prognostic or predictive factor of survival rate in SCC of the tongue, and that no clear correlation existed between this tumor marker and SCC of the tongue (Ulanovski et al., 2004). The results of research conducted by these researchers are consistent with those of the present study.

Carcinoembryonic antigen (CEA) is a glycoprotein involved in cellular adhesion. It has been evaluated in many malignancies and its levels reflect the progress or regression of the disease, which is useful in early diagnosis of the disease, its relapse, and its treatment control (Boehm et al., 2000; Bo et al., 2015). High levels of CEA are observed in patients with colon carcinoma and GI tract carcinoma (Yakabe et al., 2010).

Rafael et al (2006) analyzed salivary levels of CEA and several other tumor markers in patients with OSCC in their research, and stated that salivary concentrations of these markers increased in patients with OSCC . Abdullah S et al (2013) investigated the value of CEA in determining the prognosis of patients with colorectal and gastric cancer, and found a direct relationship between serum CEA, TNM staging, and prognosis of disease. Higher levels of CEA were associated with more advanced stages of the disease and poorer prognosis.

Xian-Jie et al. studied the diagnostic value of CEA, CA 19-9, CA 24-2, CA 72-4 serum levels for clinical diagnosis of upper gastrointestinal cancer, assessment of treatment, and prognosis of the disease. The researchers reported that studying serum levels of these tumor markers provided the best practical and economical strategy for detecting upper gastrointestinal cancer. In addition, measuring the levels of this tumor marker during follow-up periods for detecting recurrence and tumor metastasis was useful and could be used to assess disease prognosis (Xian-Jie et al., 2014).

Li-Kui et al (2015) reported that measurement of serum levels of CEA and several other tumor markers increased the sensitivity and accuracy of GI cancer diagnosis and could be useful for early diagnosis of the disease .

Considering increased levels of salivary CEA in patients with OSCC, this tumor marker can probably be used for early diagnosis of this disease in patients; however, the tumor marker erbB2 requires further research with larger sample sizes.

\section{References}

Abdullah S, Mustafa K, Gurhan B, Fatih B, Orhan A(2013). CEA and CA 19-9 are still valuable markers for the prognosis of colorectal and gastric cancer patients. Asian Pac J Cancer Prev, 14, 4289-94.

Angiero F, Sordo RD, Dessy E, et al (2008). Comparative analysis of c-erbB-2(HER-2/Neu) in squamous cell carcinoma of the tongue: does over- expression exist? And what is its correlation with traditional diagnostic parameters. J Oral Pathol Med, 37,145-50.

Bayrak M, OlmezO F, Kurt E,et al (2013). Prognostic significance of c-erbB2 over expression in patients with metastatic gastric cancer. Clin Transl Oncol, 15, 307-12

Bo H, Hui-Qing ZH, Shu-Ping X, et al (2015). Changing patterns of serum CEA and CA199 for evaluating the response to first-line chemotherapy in patients with advanced gastric adenocarcinoma. Asian Pac J Cancer Prev, 16, 3111-16.

Boehm MK, Perkins S (2000). Structural models for carcinoembryonic antigen and it's complex with the single-chain FV antibody molecule. FEBS letters. J Oral MaxillofacPathol, 1, 6-11.

Bonne NJ, Wong DT (2012). Salivary biomarker development using genomic, proteomic and metabolomics approaches. Genomic Med, 123, 75-82.

Chen CH, Tsai TL, Yang Ys, Tsai CC (2007). Studies of the serum HER-2/Neu and squamous cell carcinoma-related antigen expression in patients with oral squamous cell carcinoma. J Oral Pathol Med, 36, 83-7.

Chopra V, Thayalan D, VS J, Annasamy R, et al (2015). Salivary Her2/neu levels in differentiation of oral premalignant disorders and oral squamous cell Carcinomas. Asian Pac J Cancer Prev, 16, 5773-7.

Da Silva SD1, Cunha IW, Nishimoto IN, et al (2009). Clinicopathological significance of ubiquitin-specific protease $2 \mathrm{a}$ (USP2a), fatty acid synthase (FASN), and ErbB2 expression in oral squamous cell carcinomas. Oral Oncol, 45, 134-9.

Greenberg MS, Glick M, Ship JA (2008). Burket's Oral medicine. 11th ed. Hamilton: Bc Decker Inc, 96-171.

Jie-Xian J, Yan W, Xiao-Qin X, et al(2014). Tumor markers for diagnosis, monitoring of recurrence and prognosis in patients with upper gastrointestinal tract cancer. Asian Pac J Cancer Prev, 15, 10267-72.

Li-Kui Y, Xue-Qing S, Dong-Zhen M (2015). Value of combined detection of serum CEA, CA72-4, CA19-9 and TSGF in the Diagnosis of Gastric Cancer. Asian Pac J Cancer Prev, 16, 3867-70. 
Liu J, Duan Y (2012). Saliva potential media for disease diagnostics and monitoring. Oral Oncol, 48, 569-77.

Normanno N, De Luca A, Bianco C, et al (2006). Epidermal growth factor receptor (EGFR) signaling in cancer. Gene, 366, 2-16.

Rafael N, Gideon B, Thomas SH, Raphael F (2006). Concomitant Aanalysis of salivary tumor markers: a new diagnostic tool for oral cancer. Clin Cancer Res, 12, 3979-84.

Rautava J, Jee KJ, Miettinen PJ, et al (2008). ERBB receptors in developing, dysplastic and malignant oral epithelia. Oral Oncol, 44, 227-35.

Sardari Y, Soheil P, Akbarpoor D. (2012). Her2/Neu expression in head and neck squamous cell carcinoma patient is not significantly elevated. Asian Pac J Cancer Prev, 13, 2891-6.

Sebastian S, Settleman J, Reshkin SJ, et al (2006). The complexity of targeting EGFR signalling in cancer: from expression to turnover. Biochim Biophys Acta, 1766, 120-39.

Soulieres D, Senzer NN, Vokes EE, et al (2004). Multicenter phase II study of erlotinib, an oral epidermal growth factor receptor tyrosine kinase inhibitor, in patients with recurrent or metastatic squamous cell cancer of the head and neck. $J$ Clin Oncol, 22, 77-85.

Streckfus CF, Bigler L, Dellinger T, et al(2004). The expression of the c-erbB-2 receptor protein in glandular salivary secretions. J Oral Pathol Med, 33, 595-600.

Ulanovski D, Stern Y, Roizman P, et al (2004). Expression of EGFR and c-erbB2 as prognostic factors in cancer of the tongue. Oral Oncol, 40, 532-7.

Yakabe T, Nakafusa Y, Sumi K, et al (2010). Clinical significance of CEA and CA 19-9 in postoperative follow-up of colorectal cancer. Ann Surg Oncol, 17, 2349-56. 